\title{
Arnoldi Algorithm for the Simulation of Multidimensional Infrared Spectroscopy
}

\author{
Tomoyuki Hayashi and Shaul Mukamel \\ Department of Chemistry, Chiversit of Rochester. Rochester, NY 1+627-0216. LSA \\ Received April $1+2003$
}

\begin{abstract}
The cubic and quartic anharmonic force field of malonaldehyde is calculated using density functional theory at the B3LYP/6-31G(d.p) level, and used to simulate coherent infrared vibrational spectra. 12 normal modes are included in the simulation. and the Arnoldi method is employed for the diagonalization of the Hamiltonian. The calculated three pulse infrared signals in the $\mathbf{k}_{1}+\mathbf{k}_{2}-\mathbf{k}_{2}$ direction show signatures of the intramolecular hydrogen bond couplings between the $\mathrm{C}=\mathrm{O}$ stretch $\mathrm{H}-\mathrm{O}-\mathrm{C}$ bend and $\mathrm{O}-\mathrm{H}$ stretch vibrations.
\end{abstract}

Key Words : Amoldi algorithm, Infrared spectroscopy. Malonaldehyde. Hydrogen bonding

\section{Introduction}

Nonlinear infrared (IR) spectroscopies provide detailed information on molecular structures and their time evolution on the subpicosecond timescale. ${ }^{1,2}$ These techniques therefore provide a powerful tool for exploning the structure and proton transfer dynamics in hydrogen bonded systemts.

In previous studies. we constructed a vibrational exciton Haniltonian expanded to quartic order in a subset of internal coordinates of a metal carbonyl conplex RDC and obtained a good agreement with experiment. ${ }^{3}$ In the present work, the vibrational exciton Haniltonian is expanded in the nonnal coordinates and the Implicit Restate Annoldi Method (IRAM) is used for the diagonalization of the Haniltonian matrix. This allows us to handle a large Haniltonian matrix and describe the spectroscopy of large molecules with many vibrational coordinates.

We have calculated three pulse infrared signals of Malonaldehyde (MA). MA is a prototype for intramolecular proton transfer, and its structure and dynamics have been the focus of several experimental and theoretical investigations over the past two decades. ${ }^{+12}$ Infrared experiments show that the structure is planar and asymmetric (Figure 1)..5 Microwave spectroscopic studies of Wilsons group ${ }^{7.8}$ and others $^{69}$ suggest that proton transfer occurs via tunneling of a hydrogen atom between two oxygens. An ab Initio molecular dýnamics study at $500 \mathrm{~K}$ implied a sub ps proton transfer. ${ }^{13}$ The ab Initio potential energy surface (PES) for intramolecular proton transfer has been reported. ${ }^{11.12}$

In the present work we compute the cross peaks in infrared four-wave mixing. induced by anhamonic couplings between the $\mathrm{C}=\mathrm{O}$ stretch. $\mathrm{H}-\mathrm{O}-\mathrm{C}$ bend and $\mathrm{O}-\mathrm{H}$ stretch vibrations related to the intramolecular hyddrogen bonding.

\section{Vibrational Hamiltonian and the $A b$ Initio Force Field}

Geometry optimization and normal mode calculations of MA were executed at the B3LYP/6-31G(d.p) level using Gaussian $98 .^{13}$ The potential energy $V$ was expanded around the equilibrium geometry to quartic order in a selected set of nomal coordinates $Q_{k}$. The Hamiltonian is.

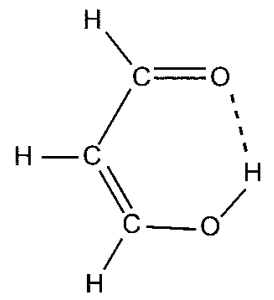

Figure 1. Equilibrium structure of MA. Intramolecular hydrogen bonding is marked by the dotted line.

$$
\begin{aligned}
& H=\sum_{i}^{3 N-6} \frac{P_{i}^{2}}{2 m_{i}}+\frac{1}{2} \sum_{i}^{3 N-6} f_{i j} Q_{i}^{2}+\frac{1}{6} \sum_{i, j, k}^{3 N-6} f_{i j k} Q_{i} Q_{j} Q_{k} \\
& +\frac{1}{24} \sum_{i, j . k . l}^{3 X_{i, k}-6} f_{l, k} Q_{3} Q_{k} Q_{l} \\
& f_{i j}=\left(\hat{\partial} V / \partial Q_{i} Q_{l}\right)_{e q} \\
& f_{i j k}=\left(\partial^{3} V / \partial Q_{j} Q_{j} Q_{k}\right)_{e q} \\
& f_{i, k l}=\left(\partial^{+} V / \partial Q_{i} Q_{j} Q_{k} Q_{l}\right)_{\epsilon q} .
\end{aligned}
$$

where () eq represents the derivatives taken at the equilibrium geometry. The expansion may be carried out using either cartesian, intemal, or nomal coordinates. ${ }^{3}$ Expressing the force field in local coordinates is most suitable for transferring parameters anong different systems. However, the kinetic energy in eq. (l) is not then diagonal and is given by the matrix:

$$
K=\dot{\mathbf{s}}^{T} \mathbf{G}^{-1} \dot{\mathbf{s}}
$$

where $s$ represents the column vector of local coordinates. $\mathbf{s}^{T}$ is its transpose. and $\mathbf{G}$ is Wilson's $\mathbf{G}$-matrix. ${ }^{14}$ The offdiagonal elements of $\mathbf{G}$ were neglected in a previous study. The normal coordinates are most suitable for the simulation of linear and nonlinear vibrational spectroscopy: using these coordinates. both the kinetic energy and the quadratic force fields are diagonal and we can cleanly separate the selected set of coordinates from all others to quadratic order. Moreover. the vibrational exciton Hamiltonian is block diagonal for normal coordinates with different șymmetries. 
The cubic and quartic anharmonic force constants were determined numerically by computing first and second derivatives of analytical quadratic force constants with 3point central difference formulas. ${ }^{15}$ The quartic expansion coefficients involving 4 different normal coordinates are small compared to the other terms and were neglected. The analy tic quadratic force constants in normal coordinates at the distorted geometry along the normal mode $Q_{k}\left(\tilde{\mathbf{F}}_{k \pm}\right)$ are obtained by

$$
\tilde{\mathbf{F}}_{k=}=\mathbf{U}_{e q}^{T} \mathbf{F}_{k \pm} \mathbf{U}_{e q} \quad(k=1, \ldots .3 N-6) .
$$

where

$$
\begin{aligned}
& \left(\tilde{\mathbf{F}}_{k==}\right)_{i j} \equiv\left(\partial^{2} V / \partial Q_{j} Q_{j}\right)_{ \pm \delta_{k}} \\
& \left(\mathbf{F}_{k=}\right)_{i j} \equiv\left(\partial^{2} V / \partial X_{r} X_{j}\right)_{\delta_{\delta_{k}}} \\
& \left(\mathbf{U}_{e q q}\right)_{l j}=\left(\partial X_{j} / \partial Q_{j}\right)_{e q} .
\end{aligned}
$$

and $\mathbf{U}_{e q,}^{T}$ represents the transposed matrix of $\mathbf{U}_{\varepsilon q \cdot .} . X_{i}$ is a cartesian coordinate. ()$_{=\dot{\delta}_{k}}$ represents the derivatives taken at the position displaced in + and - directions along the normal coordinate $Q_{k}$. The displacement $\delta$ was set to 0.02 Bohr (0.0106 A).

\section{Computing The Vibrational States}

The vibrational Hamiltonian was recast using normally ordered Bosonic creation $\left(B_{k}^{-}\right)$and anuililation $\left(B_{k}\right)$ operators and expanded in a harmonic basis set of products of local harmonic oscillators $\left(\left|m_{1} m_{2} \ldots m_{n}\right\rangle=\prod_{k}\left|m_{k}\right\rangle\right)$ following the procedure described earlier. ${ }^{3}$ The vibrational eigenstates were obtained by diagonalizing the Hamiltonian matrix with the dimension $(n+m) ! /(m ! n !)$, where $m$ is the number of modes included and $n$ is the total number of vibrational quanta. This number increases exponentially with $n$ and $m$. and only a very limited number of normal modes can be handled by standard diagonalization methods. However. we note that the Hamiltonian matrix is very sparse because all the matrix elements between two basis states which differ by more than 4 quanta vanish.

$$
H_{i j}=0 \text { if } \sum_{n=1}^{m}\left|v_{j, n}-v_{j, n}\right|>4 .
$$

where $v_{i, n}$ represents the number of vibrational quanta on the $n$ 'th normal mode in the $i$ th basis state. To save memory. the coordinate storage format was therefore used for the Hamiltonian matrix. ${ }^{16}$ In that format matrix elements are represented by three 1-dimensional array's. val( $(n n z)$ in which the value of each matrix element is stored. $i n d_{-} x(m \mathrm{mz})$ and ind_y $y(n n z)$ which represent rows and columns of these elements. Each array has the dimension of the number of nonzero elements of the matrix $(n n z)$.

Furthermore only a small number of the lowest eigenvalues and corresponding eigenvectors are needed because we are interested only in low lying vibrational states which can be excited by infrared light. Therefore the Implicit Restarted ARNOLDI Method (IRAM) ${ }^{17}$ was used for diagonalization
Table 1. Vibrational state frequencies (in $\mathrm{cm}^{-1}$ ) of $\mathrm{CO}_{2}$ calculated using the normal mode basis including all the intemal coordinates are compared with experiment. The numbers in parenthesis represent the number of quanta on the symmetric stretch, bending. and anti-symmetric stretch mode from left to right

\begin{tabular}{clrr}
\hline Exciton State & \multicolumn{1}{c}{ Eigenvector } & \multicolumn{1}{c}{ Calc. } & \multicolumn{1}{c}{ Exp. ${ }^{a}$} \\
\hline 2 & $1.0(010)$ & 651.0 & 667.4 \\
3 & $0.5(100)-0.8(020)$ & 1282.0 & 1285.4 \\
4 & $0.8(100)+0.5(020)$ & 1367.5 & 1388.2 \\
5 & $0.6(110)-0.8(030)$ & 1910.6 & 1932.5 \\
6 & $0.8(110)+0.6(030)$ & 2047.5 & 2076.9 \\
7 & $1.0(001)$ & 2361.1 & 2349.2 \\
15 & $0.4(101)-0.9(021)$ & 3610.2 & 3612.8 \\
16 & $0.8(101)+0.4(021)$ & 3707.0 & 3714.8 \\
\hline
\end{tabular}

Data from Ret. 22.

of the Hamiltonian. The Arnoldi subroutine was taken from public domain ARPACK package ${ }^{17-21}$ This algorithm is based on the iterated use of the definition of the action of $H$ on a vector $v$ to construct a sequence of vectors from a suitable first guess. The Krylov subspace spanned by these vectors is used to approximate the eigenvectors and eigenvalues. The ARPACK algorithm directly computes a set of eigenvalues and eigenvectors using the implicitly restarted Arnoldi method (IRAM). In our calculation. 25 normal modes and 4 vibrational quanta $(23.751 \times 23.751$ Hamiltonian matrix) can be included on $256 \mathrm{MB}$ RAM PC.

To test the accuracy of this method. we calculated the vibrational states of $\mathrm{CO}_{2}$ using the vibrational exciton model in the normal mode basis including all 4 internal coordinates and compared them with experiment." The B3LYP/6-31+ $G(d)$ level was employed for the calculation. The calculated and experimental frequencies of the exciton states are summarized in Table 1. The energy splittings due to the Fermi resonance calculated based on normal mode basis are $85.5 \mathrm{~cm}^{-1}$ for $\{(100),(020)\}, 137.9 \mathrm{~cm}^{-1}$ for $\{(110),(030)\}$. and $96.8 \mathrm{~cm}^{-1}$ for $\{(101),(021)\}$. and they are in very good agreement with the experimental values of $102.8 \mathrm{~cm}^{-1} .144 .4$ $\mathrm{cm}^{-1}$, and $102.0 \mathrm{~cm}^{-1}$. We found the vibrational exciton states generated from the local mode coordinates to be much less accurate.

\section{Vibrational Spectra of Malonaldehyde}

All 12 normal modes with frequency above $1000 \mathrm{~cm}^{-1}$ in $\mathrm{A}^{\prime}$ symmetry (in-plane mode) were included in our study: We adopted the following three criteria in constnucting the vibrational exciton basis. First. all states with 4 vibrational quanta are included. which is the minimum requirement for quantitative prediction of the eigenvalues and the eigenvectors of the single and double exciton states. Second. all basis states with up to 6 quanta and total energy' below 8000 $\mathrm{cm}^{-1}$ were included. Third. all states having up to fifth order coupling with the fundamental of $v_{17}(\mathrm{C}=\mathrm{O}$ stretch) are included in order to reproduce the $\mathrm{C}=\mathrm{O}$ stretch overtone $(v=$ 2) frequency more accurately. These criteria resulted in 7233 exciton states. The $7233 \times 7233$ Hamiltonian matrix was 


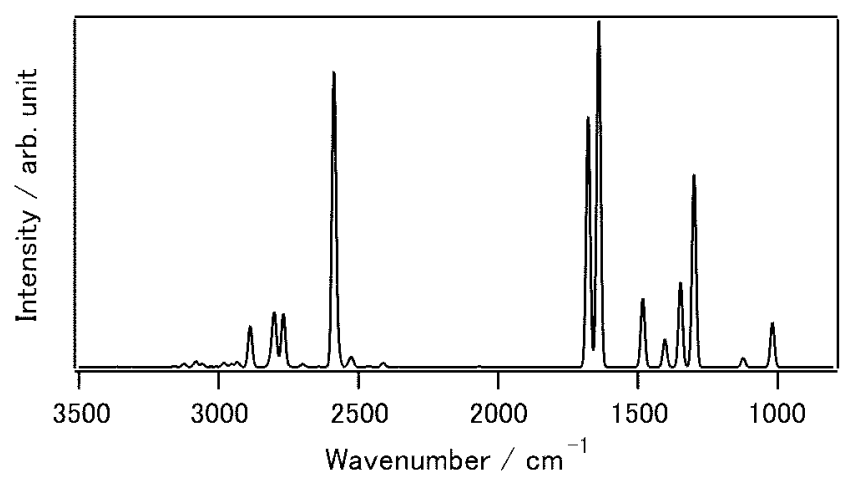

Figure 2. Simulated linear intrared absorption spectra of MA.

constructed and diagonalized to obtain the 553 eigenvalues below $5900 \mathrm{~cm}^{-1}$ and corresponding eigenvectors. The IR intensities were calculated from the dipole moment derivative with respect to the mass-weighted normal coordinates.

The calculated linear infrared absorption spectrum is shown in Figure 2. The peak frequencies are presented in Table 2 and compared with experiment. With few exceptions, the calculated peak positions from the vibrational exciton model are shifted to lower frequency compared to the hamonic ones. $v_{12}$ and $v_{13}$ have a quartic resonance and mixed with each other in the exciton calculation. The calculated $\mathrm{H}-\mathrm{O}-\mathrm{C}$ bend $\left(v_{16}\right)$ and $\mathrm{C}=\mathrm{O}$ stretch $\left(v_{17}\right)$ peaks are in good agreement with experiment. but the $\mathrm{O}-\mathrm{H}$ stretch $(19)$ frequency is lower than experiment. This is due to overestimating the cubic couplings between $v_{10}$ and the overtone of $v_{14}$. the combination band of $v_{s}$ and $v_{15} v_{11}$ and $v_{14}$, and $v_{13}$ and $v_{14}$. This can be attributed to the lack of convergence of the Taylor expansion of the PES around the equilibrium geometry. The tunneling barrier height of proton transfer is estimated to be very low $\left(42 \mathrm{~kJ}^{-\mathrm{mol}^{-1}}{ }^{23}\right)$ and quartic force field could not model this PES with a sufficient accuracy. Including a quintic $\left(f_{j i k j m}\right)$ and sextic $\left(f_{i j k m m}\right)$ force field should improve the accuracy of the peak position. However. in order to reproduce the experimental tumeling vibrational splitting

Table 2. Frequencies (in $\mathrm{cm}^{-1}$ ) obtained trom the vibrational exciton model are compared with hamonic nonmal mode calculations and with experiment

\begin{tabular}{ccccccc}
\hline \multicolumn{2}{c}{ Exciton State } & & \multicolumn{3}{c}{ Nomal Mode } & \multirow{2}{*}{ Esp. ${ }^{\sigma}$} \\
\cline { 1 - 3 } \cline { 5 - 6 } Level & Frequency & & Mode & Description & Frequency & \\
\hline 2 & 1019.0 & & $v_{8}$ & & 1018.8 & \\
3 & 1123.6 & & $v_{11}$ & & 1122.3 & 1092 \\
4 & 1299.3 & & $v_{12}$ & & 1302.3 & 1260 \\
5 & 1347.6 & & $v_{13}$ & & 1405.6 & \\
6 & 1403.6 & & $v_{14}$ & & 1419.2 & 1358 \\
7 & 1482.6 & & $v_{15}$ & & 1490.7 & 1452 \\
8 & 1640.3 & & $v_{16}$ & H-O-C bend & 1654.8 & 1593 \\
9 & 1678.0 & & $v_{15}$ & C-O stretch & 1718.1 & 1655 \\
20 & 2587.3 & & $v_{18}$ & O-H stretch & 3042.8 & 2960 \\
29 & 2801.1 & & $v_{14}$ & C-H stretch & 2991.2 & \\
41 & 3058.2 & & $v_{21}$ & C-H stretch & 3181.8 & 3100 \\
45 & 3124.0 & & $v_{21}$ & C-H stretch & 3237.6 & \\
\hline
\end{tabular}

"Data from Ref. 4.

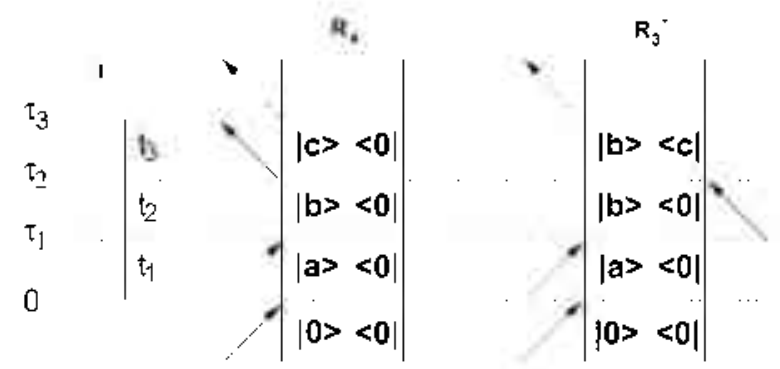

Figure 3. Double-sided Feyman diagrams deseribing the thirdorder response in the direction $\mathbf{k}_{\text {[II }}=\mathbf{k}_{1}+\mathbf{k}_{2}-\mathbf{k}_{3 .}$.

$\left(21.8 \mathrm{~cm}^{-1}\right)$, the double-well potential along the proton transfer coordinate should be modelled explicitly. ${ }^{11}$ This goes beyond the scope of this work.

We have calculated the three pulse infrared signal of MA generated in the direction $\mathbf{k}_{\text {III }}=\mathbf{k}_{1}+\mathbf{k}_{2}-\mathbf{k}_{2}$ with $\mathrm{ZZZZ}$ polarization configuration using the sum over states expression $^{24}$ and orientational factors in Eq. 13 of Reference ${ }^{25}$ The response function is given by the sum of two types of Liouville space pathways.

$$
R\left(t_{3} \cdot t_{2} \cdot t_{1}\right)=R_{4}\left(t_{3}, t_{2} \cdot t_{1}\right)-R_{3}^{*}\left(t_{3}, t_{2}, t_{1}\right)
$$

The Liouville space paths are represented by the doublesided Feynuman diagranıs shown in Figure 3 where state $a, b$ and $c$ correspond to one of the vibrational exciton states. The signal in the frequency domain is obtained by.

$$
\begin{aligned}
S\left(t_{1}, \omega_{2} . \omega_{3}\right)= & \int_{-\infty}^{\infty} d t_{3} \int_{-\infty}^{\infty} d t_{2} R\left(t_{2} \cdot t_{2}, t_{1}\right) \\
& \times \exp \left(-i\left(\omega_{3} t_{3}+\omega_{2} t_{3}\right)\right)
\end{aligned}
$$

The incident pulses were assumed to have a broad bandwidth of $-400 \mathrm{~cm}^{-1}$. A homogeneous linewidth of $3 \mathrm{~cm}^{-1}$ was taken for all transitions.

The signal for the three incident pulses tuned to 1678 . 1480 and $1480 \mathrm{~cm}^{-1}$ is displayed in Figure 4. We found 339

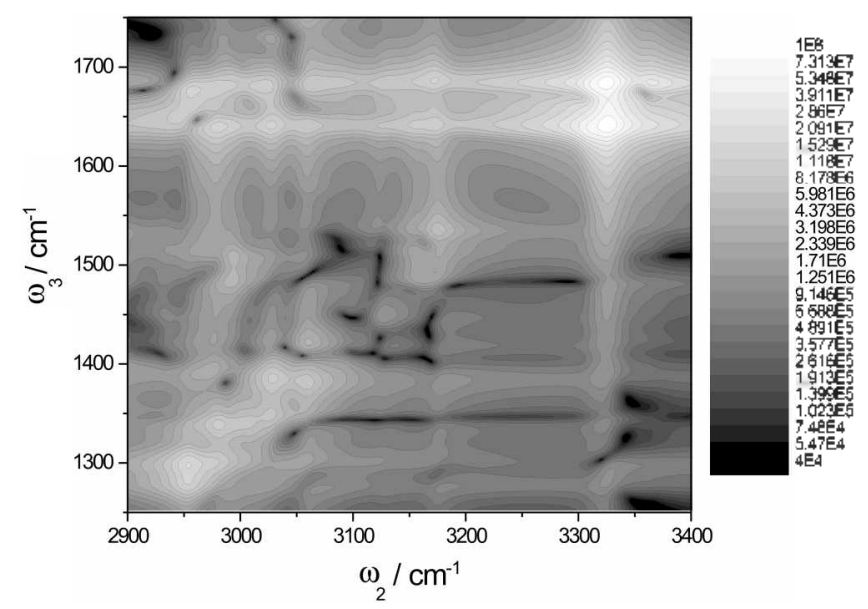

Figure 4 . Three pulse infrared signal (absolute value of $S\left(t_{1}=\right.$ $\omega_{2}, \omega_{3}$ ) (Eq. 12 ) of $\mathrm{MA}$ in the $\mathbf{k}_{3] 1}$ direction. The three 24 fsec incident pulses are tumed at 1678,1480 and $1480 \mathrm{~cm}^{-1}$ and have a band width of $420 \mathrm{~cm}^{-1}$. 
Table 3. Frequencies and corresponding Liouville space pathways (in $\mathrm{cm}^{-1}$ ) of the peaks in Figure 4 and 5 . The Liouville space pathway is specified by the diagram, $R_{4}$ or $R_{3}$ and the states $a, b$ and $c$ in Figure $3, a, b$ and $c$ comespond to one of the vibrational exciton states in Table 4

\begin{tabular}{ccccc}
\hline Peak & \multicolumn{4}{c}{ Liouville Space Pathway } \\
\cline { 2 - 5 }$\left(\omega_{2}, \omega_{3}\right)$ & Diagram & a & b & $\mathrm{c}$ \\
\hline$(3326,1640)$ & $R_{4}$ & 8 & 51 & 8 \\
$(3326,1686)$ & $R_{\pi}^{*}$ & 8 & 51 & 8 \\
$(3361,1678)$ & $R_{4}$ & 9 & 52 & 9 \\
$(3361,1683)$ & $R_{\pi}$ & 9 & 52 & 9 \\
$(2953,1678)$ & $R_{+}$ & 8 & 36 & 4 \\
$(2953,1275)$ & $R_{3}$ & 8 & 36 & 9 \\
$(3326,2587)$ & $R_{+}$ & 8 & 51 & 20 \\
$(3361,2587)$ & $R_{+}$ & 9 & 52 & 20 \\
\hline
\end{tabular}

Table 4. Frequencies (in $\mathrm{cm}^{-1}$ ) and eigenvectors of the exciton states in Table $3 . v_{v}$, represents $v$ vibrational quanta on the $v_{n}$ normal mode

\begin{tabular}{|c|c|c|}
\hline $\begin{array}{c}\text { Exciton } \\
\text { State }\end{array}$ & Frepuency & Eigenvector \\
\hline 8 & 1640.3 & $-0.94\left(1 v_{10}\right)+0.16\left(1 v_{l(k} v_{(4)}\right)+$ \\
\hline 9 & 1678.0 & $+0.93\left(1 v_{1}\right)+0.18\left(\operatorname{lv}_{1}-1 v_{19}\right)+$ \\
\hline 20 & 2587.3 & $+0.66\left(1 v_{(y)}\right)+0.30\left(2 v_{(4)}\right)+$ \\
\hline 36 & 2953.5 & $-0.71\left(1 v_{12} l_{1} v_{1}\right)-0.39\left(1 v_{0}\right)-0.34\left(1 v_{12} l v_{(6)}\right)+$ \\
\hline 51 & 3326.4 & $-0.93\left(2 v_{10}\right)-0.14\left(2 v_{10} 1 v_{(4)}\right)+$ \\
\hline 52 & 3361.5 & $+0.91\left(2 v_{1}-\right)+0.16\left(2 v_{1}-1 v_{19}\right)+$ \\
\hline
\end{tabular}

peaks generated by different Liouville space pathway's. The frequencies of peaks and corresponding Liouville space pathways are summarized in Table 3 . The frequencies and the eigenvectors of the related vibrational exciton states are given in Table 4 The strongest peaks at $\left(\omega_{3}, \omega_{3}\right)=(3326$ $\mathrm{cm}^{-1} .1640 \mathrm{~cm}^{-1}$ ) and (3326.1686) come mainly from $R_{+}$ and $R_{3}^{*}$ diagrams including the exciton states 8 and 51 . The 8 and 51 exciton states are primarily $v=1$ and $v=2$ of $v_{16}$ ( $\mathrm{H}-\mathrm{O}-\mathrm{C}$ bending mode) (Table 4), and these peaks are

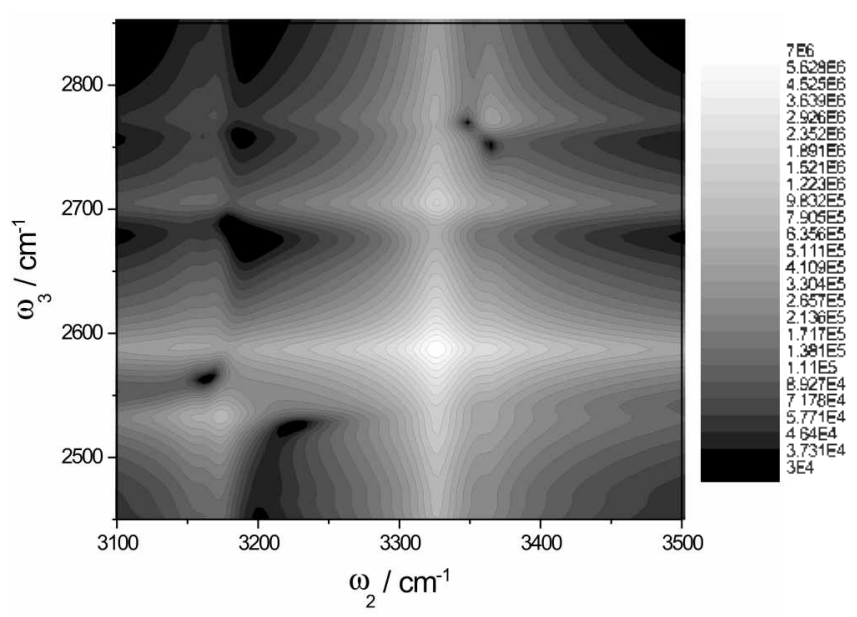

Figure 5. Three pulse infrared signal (absolute value of $S\left(t_{1}=0\right.$, $\left(\omega_{2}, \omega_{3}\right)(\mathrm{Eq} .12)$ ) of MA in the $\mathbf{k}_{\text {J] }}$ direction. The three $25 \mathrm{fsec}$ incident pulses are tuned to 1692,1669 and $774 \mathrm{~cm}^{-1}$ and have a bandwidth of $400 \mathrm{~cm}^{-1}$. generated by transitions between the fundamental and overtone of $v_{16}$. The next most intense peaks at (3361.1678) and (3361.1683) come from $R_{4}$ and $R_{3}^{*}$ diagrams involving the transitions between $v=1$ and $v=2$ of $v_{17}(\mathrm{C}=\mathrm{O}$ stretch). The weaker peaks at $(2953.1678)$ and $(2953.1275)$ correspond to $R_{4}$ and $R_{3}$ diagrams involving the transitions between $v_{17}\left(\mathrm{C}=\mathrm{O}\right.$ stretch) fundamental. $v_{1}(\mathrm{O}-\mathrm{H}$ stretch $)$ fundamental, and $v_{16}$ (H-O-C bend) fundamental. The intensities of these 2 peaks come from anharmonic (mainly cubic) couplings between $v_{17}\left(\mathrm{C}=\mathrm{O}\right.$ stretch), $v_{16}(\mathrm{H}-\mathrm{O}-\mathrm{C}$ bend). and $v_{19}(\mathrm{O}-\mathrm{H}$ stretch). There are many other peaks resulting from anharmonic couplings between different normal modes.

The $\mathbf{k}_{111}$ signal where the incident pulses are tuned at 1692 . 1669 and $774 \mathrm{~cm}^{-1}$ is displayed in Figure 5. We found 57 peaks generated by different Liouville space pathways. The strongest peak at (3326.2587) corresponds to the $R_{4}$ diagram involving transitions between the $v_{16}$ ( $\mathrm{H}-\mathrm{O}-\mathrm{C}$ bend) fundamental. overtone of $v_{15}$ (H-C-C bend), and $v_{15}(\mathrm{O}-\mathrm{H}$ stretch) fundamental. The Intensity of this peak is related to anharmonic couplings between $v_{16}$ (H-O-C bend). $v_{15}$ (H-C-C bend) and $v_{19}(\mathrm{O}-\mathrm{H}$ stretch). This peak has a side band at (3361.2587) coming from the $R_{4}$ diagram involving the transitions between fundamental $v_{17}(\mathrm{C}=\mathrm{O}$ stretch), overtone of $v_{16}(\mathrm{H}-\mathrm{O}-\mathrm{C}$ bend $)$, and $v_{1 s}(\mathrm{O}-\mathrm{H}$ stretch $)$ fundamental. The intensities of these peaks reflect anhamonic (mainly cubic) couplings between $v_{17}\left(\mathrm{C}=\mathrm{O}\right.$ stretch), $v_{16}(\mathrm{H}-\mathrm{O}-\mathrm{C}$ bend $)$, and $v_{19}(\mathrm{O}-\mathrm{H}$ stretch $)$.

\section{Discussion}

We have identified signatures of the intramolecular hydrogen bonding couplings between the $\mathrm{C}=\mathrm{O}$ stretch. $\mathrm{H}-\mathrm{O}-\mathrm{C}$ bend and $\mathrm{O}-\mathrm{H}$ stretch vibration in the $\mathbf{k}_{\| 1}$ signal. The short pulses used here generate many peaks corresponding to different Liouville pathways. Assuming a constant density of exciton states. the number of terms should be cubic in the pulse bandwidth. A $300 \mathrm{~cm}^{-1}$ bandwidth was reported in mid-infrared region by difference-frequency mixing of two visible phase-locked linearly chirped pulses in GaAs. ${ }^{26}$ and broader pulses are expected to be available in the near future.

In order to reproduce the vibrational splitting. the doublewell potential along the proton transfer coordinate and its coupling to other intramolecular coordinates should be modelled explicitly. Symmetric mode coupling (SMC) and squeezed double well potential (SQZ) which are twodimensional model potentials for proton-transfer system proposed by Takada et. al. could be used to that end. ${ }^{57}$ It is also important. especially for larger molecules. to describe highly excited low frequency modes correctly. This may be accomplished using curvilinear normal modes and local modes (CNLM) that use the Morse potential as a reference for local stretches and hamonic oscillator for the normal modes. ${ }^{28}$

Acknowledgment. We wish to thank Dr. Andrew Moran and Dr. Jens Dreyer for most useful discussions. The support of the National Science Foundation grant no. CHE-0132571 
and the support of the National Institutes of Health. grant no. 1RO1 GM59230-01A2 is gratefully acknowledged.

\section{References}

1. Mukamel, S: Hochstrasser R. M. Sipecial issure in Chem. Phus. 2001. 135.

2. Mukamel. S. Amm. Rev: Phys. 2000. 51.691

3. Moran. A. M.: Dreyer. J.: Mukamel. S. J. Chem. Phns. (in press)

4. Seliskar C. J. Hoffmann. R. E. J. Hol Spect 1982, 96, 146

5. Schiering. D. W: Katon, I. E. Appt. Spectr. 1986. $40,1049$.

6. Baba, T.: Tanaka. T: Morino. I.: Yanada. M. T.: Tanaka. K. J. Chem. Phys. 1999. 10.4131

7. Rowe. W. F.: Duerst. R. W.: Wilson. E. B. J. Am. Chem. Soc. 1977. 99. 7072 .

8. Baugheum, S. L.: Duerst. R. W: Rowe. W. F; Smith. Z.: Wilson. E. B. J.Am. Chem Soc. 1981. 103,6296.

9. Firth. D. W. Bever K Drorak M. A: Reeve, S. W.: Grushow. A.: Lepold. K. R. J. Chem. Phys. 1991. 94. 1812.

10. Wolf. K: Mikenda. W.: Nusterer. E.: Schwarz. K.: Uתbricht. C. Chem. Ew: J. 1998. 4. 1418.

11. Yagi. K.: Taketsugu. T: Hirao K. J. Chem. Phns. 2001, 115. 10647.

12. Makri, N.: Miller. W. H. J. Chen. Phns 1989.91 .4026

13. Frisch. M. I.: Trucks, G. W: Schlegel. H. B.: Seuseria. G. E: Robb, M. A.: Cheeseman, J. R.: Zakrzewski. V. G.; Montgomery. Jr.. J. A.: Stratmantr. R. E.: Burant. J. C.: Dapprich. S.: Millam. J. M.: Daniels. A. D.: Kudin. K. N.: Strain. M. C.: Farkas. O. Tomasi. J: Barone. V.: Cossi. M.: Cammi. R.: Mennucci. B.: Pomelli. C.: Adamo C.: Cliftord S.: Ochterski. J.: Petersson. G. A.: Avala. P. Y: Cui, Q.: Morokuma, K.: Malick. D. K.: Rabuck. A. D.: Raghavachari, K: Foresman, I. B.: Cioslowski. I. Ortiz, J. V: Baboul. A. G.: Stefanov. B. B.: Liu. G.: Liashenko. A.: Piskorz. P: Komaromi. I.: Gomperts. R.: Martin. R. L.: Fox. D. J: Keith. T: Al-Laham. M. A.: Peng. C. Y.: Nanayakkara. A.:
Gonzalez. C.: Challacombe, M.: Gill, P. M.: Johnson, W. B: Chen, W: Wong. M. W: Andres. J. L.: Gonzalez, C.: Head-Gordon. M.: Replogle. E. S.: Pople. I. A. Gaussian 98 nn. A. 7: Gaussian. Inc:: Pittsburgh. PA. 1998.

14. Califano. S. Tibrational States: Tohn1 Wiley and Sons: London. 1976.

15. Encyclopedia of Computational Chemismy Schlever, P. Allinger. N.: Clark. T.: Gasteiger. J: Kollman, P. A.; Schaefer III. H. A.; Schreiner. P. R.. Eds.: John Wiley and Sons: Chichester. 1998.

16. Remington. K. A.: Pozo. R. NIST Sparse BLAS Users Guide: 1996.

17. Lehoucq. R. B., Ph.D. Thesis, Rice University: Houston, Texas, 1995.

18. Sorensen. D. C. Technical Report No. TR-96-40.

19. Lehoucq. D. Y. C.: Sorensen. R. B. ARPACK Users Guide: Solution of Large Scale Eigenalue Problems by Inplicitly Restarted Anoldi Methods: 1996.

20. Maschho. K. J.: Sorensen, D. C. P. ARPACK: An Efficient Portable Large Scale Eigenvalue Package for Distributed Memory Parallel Architectures. In Applied Parallel Computing in Industrial Problems and Optimization.

21. Lecture Kotes in Computer Science: Wastiewshi. T.: Dongarra. J: Madser1. K.: Olesent. D.. Eds.: Springer-Verlag: Berlin. 1996: Vol. 1184.

22. Nakagawa, I. Tibrational Spectroscopy (in Japanese); Gakikai Shuppan Center, Tolvo. 1987.

23. Sewell. T. D.: Guo. Y.: Thompson. D. L. J. Chem. Phus. 1995. 103.8557.

24. Mukamel. S. Nonlhear Optical Spectoscopy: Osford University Press: New York, 1995.

25. Hochstrasser, R. M. Chent. Phs 2001, 266. 273.

26. Belabas. N.: Likforman. J.-P. Optics Letters $\mathbf{2 0 0 1}, 26.743$

27. Takada. S.: Nakamura. H. J. Chem. Phus. 1995. 102. 3977.

28. Zhang. Y.: Klippenstein. S. J.: Marcus. R. A.J. Chem. Phys. 1991. 94. 7319 . 